\title{
The 'Gamma-400’ space mission
}

\author{
Piero Spillantini ${ }^{1}$, on behalf of the Firenze, Pisa/Siena, Roma2, Trieste collaboration \\ INFN and University, Firenze, Italy \\ Via Sansone 1, 50019 Sesto Fiorentino (Fi) Italy \\ E-mail: spillantini@fi.infn.it
}

GAMMA-400 is a space mission included in the Russian Federal Space Program and supported by the Russian Federal Space Agency. The main characteristics of the mission are a very elliptical orbit (initial parameters: perigee $500 \mathrm{~km}$, apogee $300000 \mathrm{~km}$ ), a total mass for the scientific payload of $2600 \mathrm{~kg}$, and a power budget for the instrument of $2 \mathrm{~kW}$. The experiment is intended to improve the angular and energy resolutions obtained by other space missions for gamma and electron detections in the 0.1-3000 GeV energy range. For gamma rays of energy $100 \mathrm{GeV}$, the expected angular and energy resolution are $\approx 0.01^{\circ}$ and $\approx 1 \%$. The apparatus will consist of a finely segmented converter/tracker (made by thin tungsten layers and sensitive planes of silicon microstrip detectors), and a deep $\left(\approx 25 \mathrm{X}_{0}\right)$ homogeneous imaging calorimeter for energy measurement. On top of the Si-W converter/tracker, a light, multilayer silicon tracking detector will extend the Gamma-400 measuring capabilities to low and medium energy gamma rays in the range $50-300 \mathrm{MeV}$. The homogeneous and deep calorimeter, besides providing excellent energy resolution and rejection power, can also be used to measure cosmicray protons and nuclei entering from the sides, thus achieving a total GF for nuclei exceeding 1 $\mathrm{m}^{2} \mathrm{sr}$ and enabling the measurement, in the 10 year life foreseen for the mission, of the proton flux beyond $2 \mathrm{PeV}$ and the helium flux beyond $1 \mathrm{PeV} /$ nucleon.

10th International Conference on Large Scale Applications and Radiation Hardness of Semiconductor Detectors Firenze, Italy

July 6-8, 201

\footnotetext{
$1 \quad$ Speaker
} 


\section{Introduction}

The Gamma-400 space mission was long time ago included in the Russian Federal program for space, devoted to the observation of high energy gamma rays and the high energy electrons and positrons [1]. The experiment is intended to improve the angular and energy resolutions obtained by other space missions for gamma and electron detections in the 0.1-3000 $\mathrm{GeV}$ energy range.

The angular resolution $\left(0.01^{\circ} @ 100 \mathrm{GeV}\right)$ is obtained by a suitably stratified converter, interleaved with high resolution position measurements of the produced e-pair by microstrip $\operatorname{Si}(\mathrm{x}, \mathrm{y})$ planes.

The optimal energy resolution $(\approx 1 \%$ at $\geq 100 \mathrm{GeV})$, as well as a good identification of the electromagnetic component on the huge number of hadrons, is obtained by a deep $\left(\geq 25 \mathrm{X}^{\circ}\right)$ and highly granular BGO calorimeter equipped by several microstrip $\operatorname{Si}(\mathrm{x}, \mathrm{y})$ planes.

Italian teams participating in the PAMELA experiment were asked by the Russian colleagues and by the Russian Federal Agency for Space (Roscosmos) to consider the participation in the project of the Gamma-400 and to its mission [2]. The Italian teams accepted the invitation [3] in consideration that the characteristics of the spacecraft (supplying $2 \mathrm{~kW}$ of power to an instrument of $2600 \mathrm{~kg}$ ), the planned long duration of the mission ( $\geq 10$ years) and of the very elongated orbit (perigee $500 \mathrm{~km}$, apogee $300,000 \mathrm{~km}$ ) provide excellent opportunities to configure the mission for accomplishing, besides the gamma's astronomy and the precise electron+positron spectrum measurement, very important tasks in the domain of astrophysics, such as:

- the extension of the gamma observation capability to the 50-300 MeV range;

- the study of the proton and helium spectra up to the knee region $\left(10^{14}-10^{15} \mathrm{eV}\right)$ and beyond;

- the measurement of the individual flux of all the nuclei in cosmic ray up to the actinide group, and the energy spectra of the most abundant of them.

\section{Design of a multipurpose instrument for the Gamma-400 mission}

The scheme of the instrument proposed by the Russian groups for the high energy gammas and electron+positron spectrum measurements (fig.1) will be assumed as starting configuration for introducing the suitable modifications and improvements for producing a scheme to be simulated and possible prototypes for determining performance, mass and cost.

Preliminary simulation work was already conducted in Trieste, Firenze, Pisa/Siena and Roma2 with several configurations.

The 'tentative' configuration presently under consideration is schematically drawn in fig.2. It consists of the below described sections (from top to bottom). 


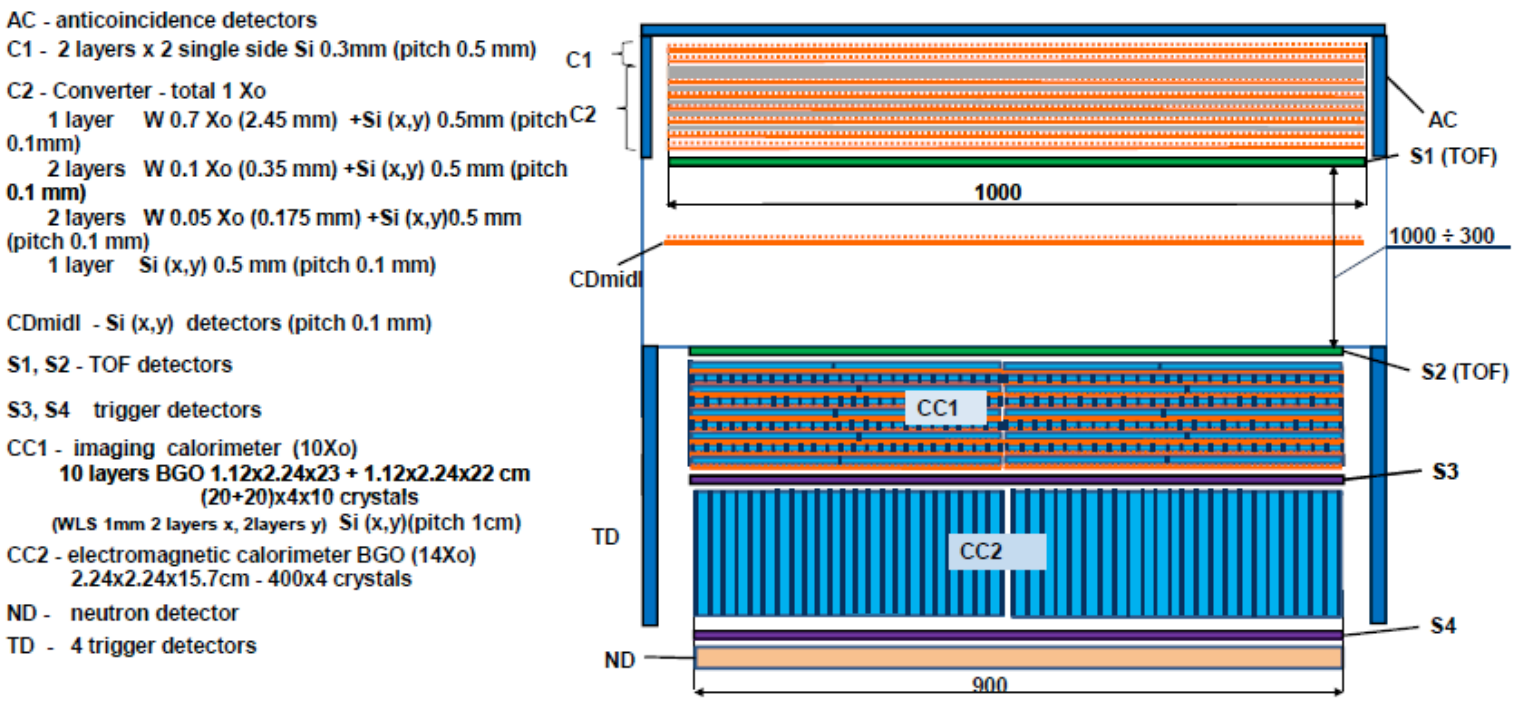

Fig. 1: Current Russian conceptual scheme of the Gamma-400 apparatus.

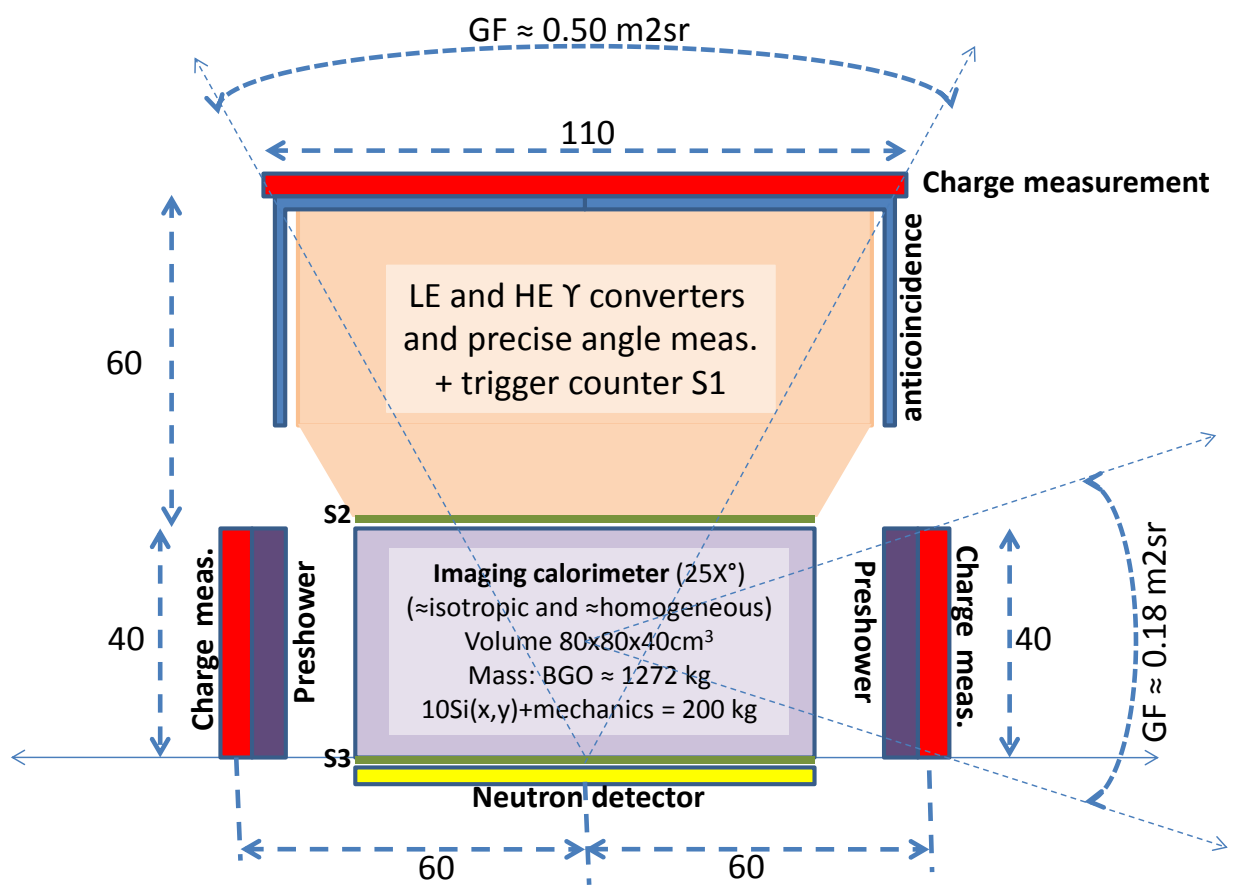

Fig. 2. Conceptual scheme of the modified Gamma-400 telescope (quotes are in $\mathrm{cm}$ ).

\subsection{Converter+tracker section}

The converter+tracker section must be optimized for a good angular resolution of the high energy gammas.

It should consist, as proposed by Russians, of $\approx 1 \mathrm{X}^{\circ}$ of tungsten stratified in 5 layers interleaving 6 microstrip $\operatorname{Si}(\mathrm{x}, \mathrm{y})$ planes with a small pitch $(\approx 0.1 \mathrm{~mm})$. The last two tungsten layers are very thin $\left(\approx 0.05 \mathrm{X}^{\circ}\right)$ for minimizing the multiple scattering of the e-pair of the gamma converting in them. A further microstrip $\operatorname{Si}(\mathrm{x}, \mathrm{y})$ plane with $0.1 \mathrm{~mm}$ pitch, placed middle way 
between the converter and the calorimeter, supplies a lever arm for an optimal measurement of the angles of the e-pair. In our scheme it could be also foreseen in front of the calorimeter, at a small distance determined by the back scattering, a further microstrip $\operatorname{Si}(\mathrm{x}, \mathrm{y})$ plane for improving the pattern and the angular precision measurement.

On top of the converter we foresee a separate low energy gamma section, constituted by a number $(\approx 20)$ of microstrip $\mathrm{Si}(\mathrm{x}, \mathrm{y})$ planes, with pitch $\approx 0.5 \mathrm{~mm}$, that will take care of the conversion of gammas in the 50-300 MeV energy range and of the measurement of the position of the produced e-pair. Simulations are under way for determining the number of planes, the gap among them, the thickness of the silicon sensors and the possible introduction of very thin tungsten layers in the package.

The converter+tracker section (fig. 2 ) could cover an area $\approx 1.1 \times 1.1 \mathrm{~m}^{2}$ and should be very compact in the vertical coordinate, possibly $\leq 0.6 \mathrm{~m}$, in order to maintain a good geometry factor $\left(\mathrm{GF} \geq 0.5 \mathrm{~m}^{2} \mathrm{sr}\right)$ for the nuclei reaching from the top the calorimeter.

A set of scintillation counters, suitably segmented, will be inserted in this section or around it for trigger and anticoincidence purpose.

\subsection{The calorimetry}

The calorimeter must be deep for ensuring a good energy resolution, and have a sufficient granularity for identifying the electromagnetic component in the hadronic shower. Furthermore the need of maximizing the GF for electrons and nuclei requires that it would be also as much as possible homogeneous and isotropic in order to accept, identify and measure energy of the charged particles impinging on it without crossing the upper section of the instrument. By equipping with suitable detectors for tracking and measuring the charge along the four sides of the calorimeter (see again fig.2) it is possible to obtain a global $\mathrm{GF} \geq 0.7 \mathrm{~m}^{2} \mathrm{sr}$, to be added to the $\geq 0.5 \mathrm{~m}^{2} \mathrm{sr}$ from the top for reaching a total GF $>1 \mathrm{~m}^{2} \mathrm{sr}$. A long duration observation with such a large GF gives, for the first time in cosmic ray research, the possibility of directly studying the chemical composition of cosmic rays in the knee region, a major achievement long pursued in last decades. This approach requires the designing and testing of an innovative configuration for the calorimeter. The material should remain the BGO proposed by Russian colleagues for the gammas (BGO is good for the value of its radiation length and also enough good for the value of its interaction length) and the structure must maintain the imaging capability, for example composing the calorimeter by many small crystals. Assuming for the calorimeter a parallelepiped shape, with $0.8 \mathrm{x} 0.8 \mathrm{~m}^{2}$ area [4] and a height of $\approx 0.40 \mathrm{~m}$ (corresponding to $\approx 25 \mathrm{X}^{\circ}$ of $\mathrm{BGO}$ and $\approx 0.12 \mathrm{~m}$ for supporting the planes and for the output of the signals from the most inner crystals) the BGO mass would be $1200 \mathrm{~kg}$, still compatible with the total mass allocated to the Gamma-400 instrument. In the most straightforward solution the sensible volume could be constituted by small cubic BGO crystals, whose granularity determines the imaging capability of the calorimeter (fig.3). The total number of small crystals depends from their side: about 14,000 for $2 X^{\circ}(2.24 \mathrm{~cm})$ side, reaching 56,000 for $1 \mathrm{X}^{\circ}(1.12 \mathrm{~cm})$ side. A more isotropic configuration would require spherical BGO crystals, which could be approximated by a trunked octahedron shape (fig.4). The advantage is that of assuring uniformity for all direction, and a work of simulation and feasibility is in progress. 


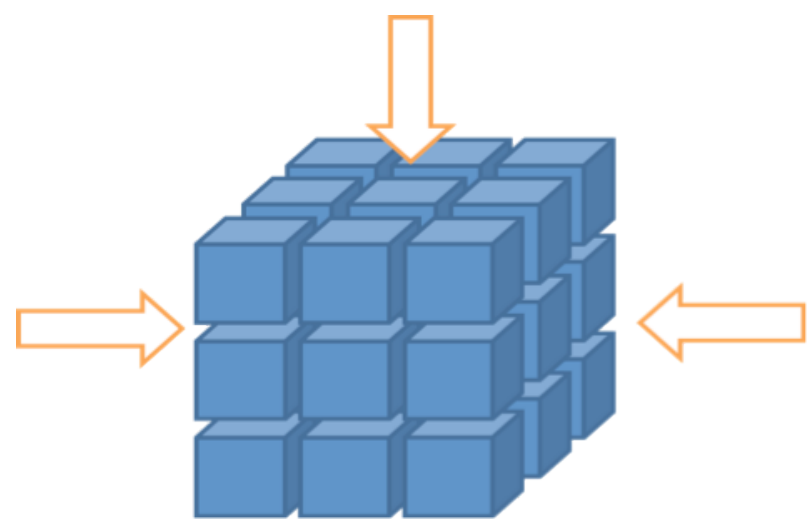

Fig.3 - Structure of a calorimeter composed by cubic small BGO crystals

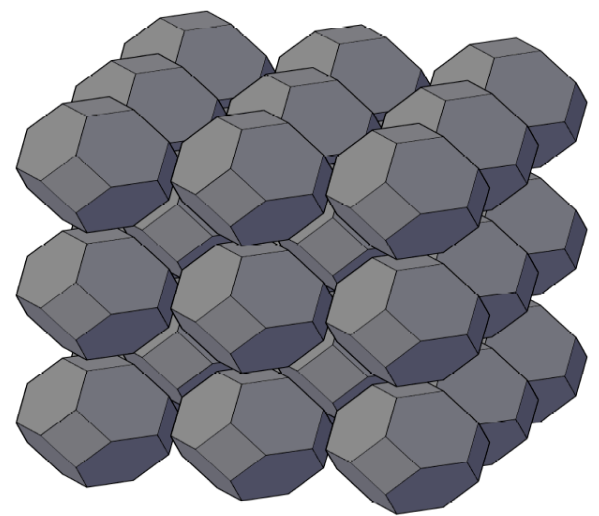

Fig.4 - An isotropic configuration would require spherical BGO crystals, which could be approximated by a trunked octahedron shape

Such a homogeneous and isotropic imaging calorimeter fulfils the original Russian requirements for the gammas, and is also capable to perform several other tasks, such as the measurement of the proton flux with a good efficiency (of the order of 30\% for above-entering particles and $50-60 \%$ for particles entering from the sides) and an effective e/p separation.

\subsection{Charge measurement}

The charge of incoming particle must be measured before its interaction with other part of the instrument: on top of the converter+tracker block for particles coming from the top and at some distance from the sides of the calorimeter for particles coming from the side. Two layers of silicon pixels are sufficient, whose granularity must deal with the back-splash from the calorimeter. Shape and area of the pixels will be determined by simulation on the final configuration of the whole instrument. Tentatively it can be assumed $1 \times 1 \mathrm{~cm}^{2}$ square pixels, whose number would be 24,000 on the top and 6,000 for each of the four sides. Moreover, the charge detectors must be equipped with a very large dynamic range electronics, enabling to measure $\mathrm{Z}$ up to the actinides without introducing ancillary counters, which (given the large dimensions) would significantly contribute to the total mass. 
For the particles incoming from sides, if the granularity of the calorimeter will be not sufficient for the identification of the electron showers on the most abundant hadronic ones, it could be required a preshower on each of the four sides of the calorimeter. Its thickness should be $\geq 1 X^{\circ}$, and should be subdivided in layers and equipped with microstrip $\operatorname{Si}(x, y)$ planes placed immediately behind the charge measurement detector.

\section{The spacecraft and the orbit}

The Gamma-400 instrument will be installed on the spacecraft 'Navigator', already used in other astrophysical missions. It is three axes stabilized and its mass is $900 \mathrm{~kg}$. The instrument will be installed on the upper plane of the spacecraft (fig.5). The solar panels and the heat radiators are at a lower level and do not interfere with the field of view of the instrument. The attitude can be maneuvered for pointing gamma sources when required. Given the isotropy of the electron and nuclei fluxes, their registration will also go on in all these phases of the mission.

The spacecraft and its instrument will be injected in a very elongated orbit, which after some months evolves to a very high circular orbit $(150-200,000 \mathrm{~km})$. It is a very favorable condition for the electric supply, but also for a long duration pointing of a given source without the dead time due to the Earth shadow.

For a so high altitude orbit the problem of the radiation belt is drastically diminished, while the flux of galactic cosmic rays will be for one order of magnitude more intense that in LEO orbit, due to the absence of the protection of the terrestrial magnetic field, but anyway limited to an integrated intensity of a few ten krad in the 10 years of duration of the mission.

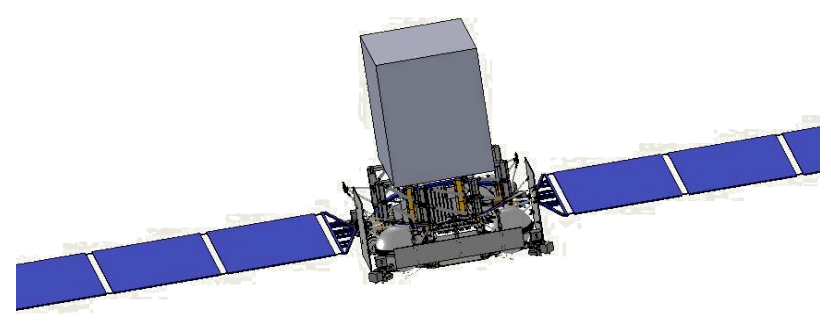

Fig.5- The Gamma-400 instrument installed on the 'Navigator' spacecraft

\section{Conclusions}

The study of the multipurpose instrument should be conducted in collaboration with the Russian colleagues, and last two years for producing at the end the design of an apparatus that could afford at an excellent competitively level the following important field of research: 
- Unprecedentedly precise measurements of the position of the sources, energy spectrum and possible time variation of high energy gamma's in the $0.1 \mathrm{GeV}-3 \mathrm{TeV}$ energy range.

- Extension of gamma's measurements down to the $50-300 \mathrm{MeV}$ region.

- High precision measurement of electron+positron spectrum (up to $>3 \mathrm{TeV}$ ).

- Measurement of the energy spectrum of protons up to $2 \mathrm{PeV}$ and beyond.

- Measurement of the energy spectrum of helium up to $1 \mathrm{PeV} / \mathrm{n}$ and beyond.

- Measurement of the individual flux of nuclei up to actinides and of the energy spectra of the most abundant ones.

The design must be optimized for the above observations in the framework of the total mass $(2600 \mathrm{~kg})$ and available electric power $(2 \mathrm{~kW})$, with the further limit on the total number of readout channels of not more than 0.5 million [5]. The limit on the number of readout channels is set mainly by consideration of the total available budget for the instrument, half already guaranteed by the Russian side and half to be asked to the Italian institutions.

The realization of the instrument could require $\approx 5$ years, and it could be launched in 2018 2019 to be operated in orbit for at least 10 years.

\section{Acknowledgements}

This contribution to the RD11 conference was presented by the author on the behalf of the INFN and University teams of Firenze, Pisa/Siena, Roma2 and Trieste. The author is grateful and acknowledges their collaboration and contributions.

\section{References and notes}

[1] V.L. Ginzburg, A.M. Galper, V.A. Kaplin, M.F. Runtso, N.P. Topchiev, M.I. Fradkin and V.G. Zverev, 'Status of the Gamma-400 project', proceedings of the $31^{\text {st }}$ ICRC, Lodz (Polony), 2009, pag.1.

[2] Minutes of the ASI-Roscosmos meeting for 'exploration of the universe', Rome, October 20, 2009.

[3] INFN, letter to dr. Serghei Saveldev, Director of the Department for foreign relations, Federal Space Agency, Moscow, Russian Federation- July 6, 2010

[4] The area $0.8 \times 0.8 \mathrm{~m}^{2}$ of the calorimeter was fixed at the Italian-Russian meeting in Moscow in November 2010. However in March 2011 Russian colleagues presented to their space agency Roscosmos the scheme of the instrument presented in fig.1, with the area of the calorimeter $0.9 \times 0.9 \mathrm{~m}^{2}$, what implies a higher mass $(1550 \mathrm{~kg}$ instead $1220 \mathrm{~kg}$ for the needed BGO), and reduced resources for the other parts to be added to the instrument. This is the main point to be clarified in next future with our Russian colleagues.

[5] The tentative distribution of the readout channels is as follows: $\approx 140 \mathrm{k}$ for the converter+tracker ( 7 microstrip $\mathrm{Si}(\mathrm{x}, \mathrm{y})$ layers, pitch $0.1 \mathrm{~mm}), 180 \mathrm{k}$ for the extension to low energy gammas (4 towers of 20 microstrip $\operatorname{Si}(\mathrm{x}, \mathrm{y})$ layers, pitch $0.5 \mathrm{~mm}$ ), $28 \mathrm{k}-110 \mathrm{~K}$ for the calorimeter (depending from the side of the BGO crystals, $2 \mathrm{X}^{\circ}$ or $1 \mathrm{X}^{\circ}$, and with two readout/crystal), $50 \mathrm{k}$ for the $1 \mathrm{x} 1 \mathrm{~cm}^{2}$ pixels for the charge measurement detectors, $10 \mathrm{k}$ for the 4 preshowers (area $0.4 \times 0.8 \mathrm{~m}^{2} /$ each, 4 microstrip $\mathrm{Si}(\mathrm{x}, \mathrm{y})$ layers, pitch $0.5 \mathrm{~mm}$ ). The total would range between $408 \mathrm{k}$ and $490 \mathrm{k}$. 